\title{
THE CALOGERO BOUND FOR NON-ZERO ANGULAR MOMENTUM
}

\author{
K. Chadan \\ Laboratoire de Physique Théorique et Hautes Energies* \\ Université de Paris XI, bâtiment 211, 91405 Orsay Cedex, France
}

A. Martin and J. Stubbe

CERN, Theory Division, 1211 Geneva 23, Switzerland

\begin{abstract}
We show that the "Calogero" bound on the number of bound states in an attractive monotonous potential is not optimal for a strictly positive angular momentum $\ell$ and propose a new bound including an extra additive term. It is: $N_{\ell}(V)<\frac{2}{\pi} \int_{0}^{\infty} \sqrt{|V(r)|} d r+1-\sqrt{1+\left(\frac{2}{\pi}\right)^{2} \ell(\ell+1)}$. From this new bound it is possible to obtain a bound on the total number of bound states for arbitrary angular momentum.

We also investigate the situation for $-\frac{1}{2} \leq \ell<0$ and give a bound under the condition that $r^{2} V(r)$ has a single extremum. Consequences for zero angular momentum bound states in two dimensions are discussed.
\end{abstract}

CERN-TH.7158/94

LPTHE Orsay $94 / 08$

January 1994

* Laboratoire associé au Centre National de la Recherche Scientifique - URA 63 


\section{Introduction}

Many years ago, Calogero ${ }^{(1)}$ has proposed a bound on the number of bound states of a particle obeying the Schrödinger equation, submitted to a potential which is negative, monotonous increasing. This bound, for angular momentum $\ell=0$, is, in units where $2 m$ ( $m$ mass of the particle) $=\hbar=1$ is

$$
N_{0}(V) \leq \frac{2}{\pi} \int_{0}^{\infty} \sqrt{-V(r)} d r
$$

The constant in (1) is easily seen to be the best possible one, since the bound is saturated by a square well

$$
\left.\begin{array}{cc}
V=-V_{0} & \text { for } \quad V<R \\
V=0 & \text { for } \quad V \geq R
\end{array}\right\}
$$

with $V_{0} R^{2}=\left(\frac{\pi}{2}\right)^{2}$.

Furthermore, by taking a finite succession of adjacent square wells with ratios between successive strengths arbitrarily small, one can build examples in which the left hand-side of (1) is $n$ and the right hand-side $n+\varepsilon, \varepsilon$ arbitrarily small.

For general angular momentum $\ell>0$ the Hamiltonian is

$$
-\frac{d^{2}}{d r^{2}}+\frac{\ell(\ell+1)}{r^{2}}+V(r)
$$

and it is obvious that the bound (1) also holds for arbitrary $\ell$, because of the positivity of the centrifugal term in (3). Hence, under the same assumptions on $V$, we have

$$
N_{\ell}(V) \leq \frac{2}{\pi} \int_{0}^{\infty} \sqrt{-V(r)} d r \quad, \quad \ell \geq 0
$$

The question is now to know whether this bound is optimal. In Reed and Simon $^{(2)}$, vol. IV, p. 97 it is stated : 
"Bargmann's bound, Calogero bound, $\cdots$, are "best possible" in the sense that there exist potentials with $N_{\ell}(V)$ (or $\ell_{\max }(V)$ ) any preassigned integer and with the integral on the right hand-side arbitrarily close to $N_{\ell}(V)$ or $\ell_{\max }(V)$. Thus the constants $(2 \ell+1)^{-1}, 2 / \pi$ cannot be replaced with smaller constants ...".

For the Bargmann bound we agree that this is perfectly correct ${ }^{(3)}$. For the Calogero bound we are not certain of this and in fact we shall explain below why we have very serious doubts.

The reason for these doubts will be explained below, but we would like to stress that independently of this question there are (at least !) two ways for a bound to be optimal. One, which is the most commonly used, is that a bound $B_{\ell}(V)$ is said to be optimal if

$$
\sup \frac{N_{\ell}(V)}{B_{\ell}(V)}=1
$$

where the sup is taken over all $V$ 's acceptable.

The other way is to have

$$
\sup \left[N_{\ell}(V)-B_{\ell}(V)\right]=0
$$

For the Bargmann bound ${ }^{(3)}$

$$
N_{\ell}(V)<\frac{1}{2 \ell+1} \int r|V(r)| d r
$$

is optimal in both ways, i.e. (5) and (6), and the generalizations involving integrals of powers of the potential by Glaser et $\mathrm{al}^{(4)}$ are also optimal in both ways (we ask the reader to trust us for that).

Concerning the Calogero bound, we shall prove that (6) is certainly not true and replace (4) by another, more accurate bound, containing an extra additive term depending on $\ell$. This term itself is not strictly optimal but, in a sense which will be explained in the next section, optimal for very large $\ell$.

Let us return now to the question of optimality of the Calogero bound for $\ell>0$ in the "ordinary" sense i.e. (5). 
First of all, we remark that the constant in (4), which is $2 / \pi$ cannot be "wrong" by more than a factor 2 , because

$$
\sup \frac{N_{\ell}(V)}{\int \sqrt{-V d r}} \geq \frac{1}{\pi}
$$

We know that if we take a potential $\lambda V, \lambda \rightarrow \infty$, the number of bound states, for large $\lambda$ reaches the "semi classical limit" at least for $\ell=0^{(5)}$, i.e.

$$
\frac{N_{0}(\lambda V)}{\lambda^{1 / 2}} \rightarrow \frac{1}{\pi} \int \sqrt{\left|V_{-}\right|} d r
$$

where $V_{-}$is the negative part of the potential.

For non zero angular momentum we can replace the interval $0<r<\infty$ by $0<r_{1}<r<r_{2}$ with Dirichlet boundary conditions on $r_{1}$ and $r_{2}$. This can only diminish the number of bound states. Then, in $r_{1}<r<r_{2}$ the centrifugal term gets negligible in the effective potential $\lambda V(r)+\frac{\ell(\ell+1)}{r^{2}}$ for sufficiently large $\lambda$, and $\sqrt{-\lambda V-\frac{\ell(\ell+1)}{r^{2}}} \simeq \sqrt{-\lambda V}$.

The conclusion is that we cannot improve the $2 / \pi$ in (4) by more than replacing by $1 / \pi$. Yet, we believe that $2 / \pi$ is not the optimum and we shall explain why. The number of bound state in a potential is known to be equal to the number of nodes of the zero energy wave function with the boundary condition $u(r=0)=0$. The $\ell \neq 0$ Schrödinger equation

$$
\left(-\frac{d^{2}}{d r^{2}}+\frac{\ell(\ell+1)}{r^{2}}+V(r)\right) u(r)=0
$$

can be transformed, by a change of variables and change of functions into an equation looking like a zero angular momentum wave equation :

$$
\left(-\frac{d^{2}}{d Z^{2}}+W(Z)\right) U(Z)=0
$$

where $W(Z)=\frac{r^{-4 \ell} V(r)}{(2 \ell+1)^{2}}$

$$
\begin{gathered}
Z=r^{1+2 \ell} \\
U(Z)=r^{\ell} u(r)
\end{gathered}
$$


if $-V(r)$ is monotonous decreasing

$-W(Z)$ is also monotonous decreasing and hence we can apply the Calogero bound

$$
\begin{aligned}
N_{\ell}(V) & <\frac{2}{\pi} \int \sqrt{-W(Z)} d Z \\
& =\frac{2}{\pi} \int \sqrt{-V(r)} d r .
\end{aligned}
$$

This is not a surprise! However we know that the conditions for the saturation of the Calogero bound for $\ell=0$ are, among others, that $V(r)$ be constant between a zero of $u(r)$ and the next maximum of $|u(r)|$. Returning to $W(Z)$ we see that these conditions cannot be met for $|W(Z)|=r^{-4 \ell}|V(r)| .|W(Z)|$ for monotonous $V$ is strictly decreasing.

The class $K_{\ell}$ of potentials for which the Calogero bound for $N_{\ell}(V)$ is saturated is

$$
K_{\ell}\left\{\begin{array}{c}
V(r)<0 \\
\frac{d V(r)}{d r} \geq 4 \ell \frac{V(r)}{r}
\end{array}\right.
$$

this class is bigger than $K_{0}$, for $\ell>0$. In fact, bounds for potentials of this class have been already considered by one of us (K.C.) and R. Kobayashi ${ }^{(6)}$. Take for instance $\ell=1$ :

$$
K_{1}\left\{\begin{array}{c}
V(r)<0 \\
\frac{d V(r)}{d r} \geq 4 \frac{V}{r}
\end{array}\right.
$$

a potential of this class, is

$$
\left\{\begin{array}{cll}
V=-\lambda r^{4} & \text { for } & r<R \\
V=0 & \text { for } & r>R
\end{array} .\right.
$$

From the Calogero sufficient condition for the existence of an $\ell=0$ bound state $^{(7)}$

$$
V<0, \frac{1}{R} \int_{0}^{R^{\prime}} r^{2}|V| d r+\int_{R^{\prime}}^{\infty} r|V| d r>1
$$

we see that we shall certainly have an $\ell=0$ bound state if

$$
\lambda R^{6}=6
$$


while the Calogero condition (eq. (1)) for $\ell=0$ would guarantee the absence of bound state for $\ell=0$ if

$$
\lambda R^{6}<\left(\frac{3 \pi}{2}\right)^{2} \simeq 22.2 .
$$

This is a (not unexpected) contradiction. In other words, the potentials of the bigger class $K_{1}$, saturating the Calogero bound for $\ell=1$, can violate the Calogero bound for $\ell=0$ and we have every reason to expect that the smaller class $K_{0}$ which saturates the Calogero bound for $\ell=0$ will not saturate the Calogero bound for $\ell=1$.

\section{Improved Calogero bound for $\ell>0$}

As we said in the Introduction, we shall try to get a bound of the form

$$
N_{\ell}(V)<\frac{2}{\pi} \int_{0}^{\infty} \sqrt{-V(r)} d r+C_{\ell}
$$

with $C_{\ell}<0$ for $\ell>0$. We shall proceed by successive approximations. Let us define

$$
I(r)=\int_{0}^{r} \sqrt{-V\left(r^{\prime}\right)} d r^{\prime}
$$

Because of the monotonicity of $|V|$, we have

$$
\left.\begin{array}{c}
I(r)>r \sqrt{|V(r)|} \\
\text { or }|V(r)|<\left[\frac{I(r)}{r}\right]^{2}<\frac{(I(\infty))^{2}}{r^{2}}
\end{array}\right\}
$$

This means that if we combine this inequality with the familiar operator inequality

$$
-\frac{d^{2}}{d r^{2}}>\frac{1}{4 r^{2}}
$$

we get a lower bound for the Hamiltonian :

$$
H_{\ell}>\frac{(\ell+1 / 2)^{2}-(I(\infty))^{2}}{r^{2}} .
$$


Hence we conclude that there is no bound state with angular momentum $\ell$ if :

$$
\int_{0}^{\infty} \sqrt{|V(r)|} d r<\ell+1 / 2
$$

If $\int_{0}^{\infty} \sqrt{|V(r)|} d r$ exceeds $\ell+1 / 2$ there exists $R_{\ell}$ such that

$$
\int_{0}^{R_{\ell}} \sqrt{|V(r)|} d r=I\left(R_{\ell}\right)=\ell+1 / 2
$$

In the interval $R_{\ell}<r<\infty$, with a Dirichlet boundary condition at $r=R_{\ell}$ the number of bound states is certainly less than

$$
\frac{2}{\pi} \int_{R_{\ell}}^{\infty} \sqrt{|V(r)|} d r
$$

since throwing away the centrifugal term increases the number of bound states and makes the effective potential monotonous.

Returning now to the full interval $0<r<\infty$, we see that the zero energy wave function has no node for $r<R_{\ell}$ and has at most

$$
\frac{2}{\pi} \int_{R_{\ell}}^{\infty} \sqrt{|V(r)|} d r+1
$$

nodes in $R_{\ell} \leq r<\infty$, since changing the boundary conditions can only increase the number of nodes by one unit. Hence (21) represents a bound on the number of bound states and, using (20), we get

$$
N_{\ell}(V)<1+\frac{2}{\pi}\left[\int_{0}^{\infty} \sqrt{|V(r)|} d r-(\ell+1 / 2)\right] .
$$

This bound has the defect to exceed by $1-1 / \pi$ the original Calogero bound for $\ell=0$. However this can be remedied by a slightly more subtle argument.

From (17), we have

$$
\left(\frac{1}{r}\right)^{2}>\frac{|V(r)|}{(I(R))^{2}} \quad \text { for } \quad 0<r<R
$$


and, hence

$$
V_{e f f}=\frac{\ell(\ell+1)}{r^{2}}+V(r)>-|V(r)|\left[1-\frac{\ell(\ell+1)}{(I(R))^{2}}\right]
$$

the right hand-side of (23) is again a montonous potential. Hence the wave function will be nodeless in $0<r<R$ if

$$
\frac{1}{\pi} \int_{0}^{R} \sqrt{|V(r)|} d r \sqrt{1-\frac{\ell(\ell+1)}{(I(R))^{2}}}=1
$$

or

$$
I(R)=\sqrt{\left(\frac{\pi}{2}\right)^{2}+\ell(\ell+1)}
$$

the rest of the argument is similar to what we have done before ; and we get

$$
N_{\ell}(V)<\frac{2}{\pi} \int_{0}^{\infty} \sqrt{|V(r)|} d r+1-\sqrt{1+\left(\frac{2}{\pi}\right)^{2} \ell(\ell+1)}
$$

this bound coincides with the Calogero bound for $\ell=0$, has the same qualitative dependence as (22) for large $\ell$, and is always better.

Let us show now that this bound is almost optimal in a certain way for large $\ell$. Specifically let us look at condition (19) and compare with the case of a square well, of depth $-k^{2}$ and radius $R$. The wave function inside the square well is

$$
u=\sqrt{k r} J_{\ell+1 / 2}(k r),
$$

where $J_{\nu}$ is a Bessel function of order $\nu$.

Since the external wave function for a zero energy bound state is

$$
u=C r^{-\ell}
$$

the condition for a zero energy bound state is

$$
\frac{J_{\ell+1 / 2}^{\prime}(X)}{J_{\ell+1 / 2}(X)}=-\frac{\ell+1 / 2}{X}
$$


where $X=k R$. $X$ is clearly located between the first maximum $X_{\max }$, and the first zero $X_{0}$ of $J_{\ell+1 / 2}$. It is well known that ${ }^{(8)}$ for large $\ell$,

$$
\begin{aligned}
& X_{\max }-\ell=0\left(\ell^{1 / 3}\right) \\
& X_{0}-\ell=0\left(\ell^{1 / 3}\right)
\end{aligned}
$$

while condition (19) gives

$$
\int \sqrt{|V|} d r=k R<\ell+1 / 2
$$

so that the relative error committed by using (19) (or (25)) is of the order of $\ell^{-2 / 3}$.

Let us show now that by using (22) we can get a bound on the total number of bound states with a weight $(2 \ell+1)$ for each angular momentum which has the correct coupling constant dependence. For simplicity we use (22) (this makes very little difference!)

$$
\begin{aligned}
N_{\text {tot }}(V) & =\sum_{L}(2 \ell+1) N_{\ell}(V) \\
& \leq \sum_{0}^{L}(2 \ell+1)\left[1+\frac{2}{\pi}\left(\int_{0}^{\infty} \sqrt{|V|} d r-(\ell+1 / 2)\right)\right]
\end{aligned}
$$

with

$$
L=\left[\int_{0}^{\infty} \sqrt{|V|} d r-1 / 2\right]
$$

where the symbol [ designates the integral part of a number.

A crude estimate of the bound on $N_{\text {Tot }}$ can be obtained by replacing sums by integrals. This gives

$$
N_{\text {tot }}(V) \leq \frac{2}{3 \pi}\left(\int_{0}^{\infty} \sqrt{|V|} d r\right)^{3}+\text { corrections }
$$

This means that for a potential $\lambda V$ the bound behaves like $\lambda^{3 / 2}$ for large $\lambda$. 
In fact, after some elementary but tedious work, one can find a strict bound of a relatively simple form :

$$
N_{t o t}(V)<\frac{2}{3 \pi}\left[\frac{\pi}{2}+\int_{0}^{\infty} \sqrt{|V|} d r\right]^{3}
$$

It may be of some interest to compare this bound with the bound obtained by Lieb $^{(9)}$ and Cwikel ${ }^{(10)}$

$$
N_{\text {tot }}(V)<\text { const } \times \int\left|V_{-}\right|^{3 / 2} d^{3} x
$$

Concerning the constant, we know that the best possible constant one can hope for corresponds to the one bound state case. It would be

$$
N_{t o t}(V)<\frac{4}{3 \sqrt{3} \pi^{2}} \int\left|V_{-}\right|^{3 / 2} d^{3} x
$$

At present the latest proved bound is :

$$
N_{\text {tot }}(V)<0.116 \int\left|V_{-}\right|^{3 / 2} d^{3} x
$$

If $V$ is negative and monotonous increasing, the bound (30) implies also a bound of the form (28). Using inequality (17) we have

$$
\int_{0}^{\infty} r^{2}(V(r))^{3 / 2} d r<\int_{0}^{\infty}(I(r))^{2} d I(r)=\frac{1}{3}\left(\int_{0}^{\infty} \sqrt{|V|(r)} d r\right)^{3}
$$

Inequality (32) is saturated for a pure square well.

If we believe (30) we get, for a monotonous spherically symmetric potential, by combining (30) and (32) :

$$
N_{t o t}(V)<\frac{16}{9 \sqrt{3} \pi}\left(\int \sqrt{|V|} d r\right)^{3}
$$

the constant in front is, numerically, 
while the one in $(28)$ is

$$
0.212 \text {. }
$$

This is not a contradiction since the potential which saturates (30) is not a square well. It is nevertheless gratifying that the constants are of the same order of magnitude.

\section{The Calogero bound for $-\frac{1}{2}<\ell \leq 0$}

For a "regular" potential it is well known that the angular momentum can be continued down to $\ell=-1 / 2$. "Regular" means in a loose sense "less singular than $-r^{-2}$ ". This could be strictly

$$
\lim _{r \rightarrow 0} r^{2} V(r)=0
$$

but one can also use integral conditions such as

$$
\begin{gathered}
\int_{0}^{\infty}|V|^{\nu} r^{2 \nu-1} d r<\infty, \\
\nu \geq 1 / 2 .
\end{gathered}
$$

The requirement that the integral of $\sqrt{|V|}$ converges, which is implicit in the Calogero bound is precisely one of these conditions, with $\nu=1 / 2$.

First we shall try to go as far as possible, using the same assumptions as in the previous section, namely

$$
\begin{aligned}
& \text { i) } V<0 \quad V^{\prime}>0 \\
& \text { ii) } \int_{0}^{\infty} \sqrt{|V|} d r<\infty .
\end{aligned}
$$

The technique which has given the best results, so far is to start from the Bargmann bound (7), which is valid as well for $-1 / 2<\ell \leq 0$ as for $\ell \geq 0$. The Bargmann bound implies that there is no bound state if

$$
\frac{1}{2 \ell+1} \int_{0}^{\infty} r|V(r)| d r<1
$$


and, using inequality (17) in the same way as in the end of section II, with $I(r)$ defined by (16) :

$$
\int_{0}^{\infty} r|V(r)| d r<\int_{0}^{\infty} I(r) \sqrt{|V(r)|} d r=\frac{1}{2}\left[\int_{0}^{\infty} \sqrt{|V(r)|} d r\right]^{2} .
$$

Therefore

$$
N_{\ell}(V)=0 \quad \text { if } \frac{1}{2 \sqrt{\ell+1 / 2}} \int_{0}^{\infty} \sqrt{|V(r)|} d r<1
$$

Suppose now that we have a potential with $N$ bound states. The zero energy wave function has $N$ zeros outside the origin. Let $r_{k}$ and $r_{k+1}$ be two successive zeros. In this interval the effective potential satisfies the inequality :

$$
\left\{\begin{array}{c}
V_{e f f}=V(r)+\frac{\ell(\ell+1)}{r^{2}}>V(r)+\frac{\ell(\ell+1)}{\left(r-r_{k}\right)^{2}} \\
\text { for } \ell \text { NEGATIVE }
\end{array}\right.
$$

Hence the potential $V(r)$ with angular momentum $\ell$ relative to the new origin $r_{k}$ and a Dirichlet boundary condition at $r_{k}$ must admit a bound state because of the monotonicity of bound state energies with respect to the potential. Hence

$$
\int_{r_{k}}^{r_{k+1}} \sqrt{|V(r)|} d r>2 \sqrt{\ell+1 / 2}
$$

and, adding up all contributions

$$
\begin{gathered}
N_{\ell}(V)<\frac{1}{2 \sqrt{\ell+1 / 2)}} \int_{0}^{\infty} \sqrt{|V(r)|} d r \\
\text { for } \ell \leq 0
\end{gathered}
$$

It is worth noticing that for $\ell=0$ this gives back the Calogero bound with a small deterioration of the constant $: \frac{2}{\pi} \simeq 0.6366 \ldots$ is replaced by $\sqrt{2} / 2 \simeq 0.707 \cdots$.

This bound is singular for $\ell \rightarrow-1 / 2$ and indeed it should be singular because $\ell=-1 / 2$ is equivalent to a zero angular momentum state in two dimensions for which there is always a bound state for an arbitrarily small attractive potential. 
However we believe that the bound (37) is too singular. The only result we could prove in this direction is rather weak. It is

$$
\left\{\begin{array}{c}
\lim _{\ell \rightarrow-1 / 2} \sqrt{2 \ell+1} N_{\ell}(V)=0 \\
\text { if } \int \sqrt{|V|} \text { converges and } V \text { is monotonous }
\end{array}\right. \text {. }
$$

We split the interval $0<r<\infty$ in three pieces $0<r_{1}<r_{2}<\infty$ in $0-r_{1}$ and $r_{2}-\infty$ we apply (37) in $r_{1} r_{2}$ we use the effective potential. We get, adding two units to correct for the imposition of Dirichlet boundary conditions at $r_{1}$ and $r_{2}$

$$
\begin{gathered}
N_{\ell}(V)<\frac{1}{2 \sqrt{\ell+1 / 2}}\left[\int_{0}^{r_{1}} \sqrt{|V|} d r+\int_{r_{2}}^{\infty} \sqrt{|V|} d r\right]+2 \\
+\int_{r_{1}}^{r_{2}} \sqrt{|V|+\frac{|\ell(\ell+1)|}{r^{2}}} d r
\end{gathered}
$$

and using $\sqrt{A+B}<\sqrt{A}+\sqrt{B}$ for $A, B$ positive,

$$
\begin{aligned}
& N_{\ell}(V) \sqrt{\ell+1 / 2}<\frac{1}{2}\left[\int_{0}^{r_{1}} \sqrt{|V|} d r+\int_{r_{2}}^{\infty} \sqrt{|V|} d r\right] \\
& +\sqrt{\ell+1 / 2}\left[2+\int_{0}^{\infty} \sqrt{|V|} d r+\sqrt{|\ell(\ell+1)|} \log \frac{r_{2}}{r_{1}}\right]
\end{aligned}
$$

by choosing $r_{1}$ and $r_{2}$ sufficiently close to zero and infinity respectively we can make the first bracket arbitrarily small and then the second term arbitrarily small by taking $\ell$ sufficiently close to $-1 / 2$. This proves our assertion.

We could not really go further without changing our assumption on $V$. Our next result is the following :

Theorem if $V$ is negative, and if $r^{2} V(r)$ has a single minimum in $0<r<\infty$, one has

$$
N_{-1 / 2}(V)<1+\frac{2}{\pi} \int_{0}^{\infty} \sqrt{|V|} d r
$$

Naturally, monotonicity in $\ell$ implies that the inequality also holds for arbitrary $\ell \geq-1 / 2$. 
All one has to do is to make the change of variables (11) in the limit $\ell=-1 / 2$. This gives, if

$$
\left.\begin{array}{c}
u^{\prime \prime}-\frac{1}{4 r^{2}} u+V u=0 \\
\phi(s)=r^{1 / 2} u(r) \\
s=\ell n r \\
\phi^{\prime \prime}(s)+W(s) \phi(s)=0 \\
W(s)=V(r) r^{2}
\end{array}\right\}
$$

the assumed convergence of $\int_{0}^{\infty} \sqrt{|V(r)|} d r$ implies

$$
\lim \inf r^{2}|V(r)|=0
$$

both for $r \rightarrow 0$ and $r \rightarrow \infty$.

The simplest assumption one can make is that $r^{2}|V(r)|$ has a single maximum at $r=r_{0}$ i.e. $W(s)$ has a single maximum at $s=s_{0}$. Imposing a Dirichlet boundary condition at $s=s_{0}$ one can use the standard Calogero bound to get a bound on the number of nodes of the wave function to the left and to the right of $s_{0}$ :

$$
\frac{2}{\pi} \int_{-\infty}^{s_{0}} \sqrt{|W(s)|} d s \text { and } \frac{2}{\pi} \int_{s_{0}}^{\infty} \sqrt{|W(s)|} d s
$$

removing carefully the Dirichlet boundary condition leads to add one unit to get a bound on the number of bound states. Then making the change of variables back to $r$ one gets inequality (39).

Inequality (39) is satisfactory because of the presence of an extra unit contribution which is unavoidable if the Hamiltonian is interpreted as that of a zero angular momentum particle in two dimensions. What is less satisfactory is that we have been forced to change our assumptions on $V$. At the present time we do not know if this is unavoidable. 


\section{Acknowledgments}

One of us (K. C.) would like to thank Professor Chaichian for warm hospitality at the Research Institute for Theoretical Physics in Helsinki, where part of this work was done.

\section{$\underline{\text { References }}$}

(1) F. Calogero : Comm. Math. Phys. 1, 1965, p. 80.

(2) M. Reed and B. Simon : Methods of Modern Mathematical Physics, Academic Press, New York, 1979.

(3) V. Bargmann : Proc. Nat. Acad. Sci. USA $\underline{38}, 1952$, p. 961.

J. Schwinger : Proc. Nat. Acad. Sci. USA 47, 1961, p. 122.

(4) V. Glaser, H. Grosse, A. Martin and W. Thirring : in Studies in Mathematical Physics, E. Lieb, B. Simon and A. Wightman Eds., Princeton University Press, 1976, p. 169.

(5) K. Chadan : Nuovo Cimento 58A, 1968, p. 191.

(6) K. Chadan and R. Kobayashi : in Rigorous Methods in Particle physics, S. Ciulli, F. Scheck and W. Thirring Eds., Springer-Verlag, 1990, p. 14.

(7) F. Calogero : J. Math. Phys. $\underline{6}$, 1965, p. 161.

(8) M. Abramovitz and I. Stegun: Handbook of Mathematical Functions, Dover, New York, 1982.

(9) E. Lieb : Proc. AMS Conf. Honolulu, 1979, S. H. Gould ed., AMS, Providence.

(10) M. Cwikel : Ann. Math. 106, 1977, p. 93. 